phys. stat. sol. (b) 224, No. 1, 191-194 (2001)

\title{
Luminescence of CdS Nanoparticles Doped with Mn
}

\author{
M. V. Artemyev (a), L. I. Gurinovich (b), A. P. Stupak (b), \\ and S. V. GAPONENKO $\left.\left.{ }^{1}\right)^{*}\right)(b)$ \\ (a) Institute for Physico-Chemical Problems, Belarussian State University, \\ Leningradskaya Str. 14, 220080 Minsk, Belarus \\ Fax: +375172264696
}

(b) Institute of Molecular and Atomic Physics, National Academy of Sciences, F. Skaryna Ave. 70, 220072 Minsk, Belarus

Fax: +375172840030

(Received July 31, 2000; accepted October 2, 2000)

Subject classification: 78.55.Et; 78.67.Bf; S8.11

Sol-gel films containing manganese-doped CdS nanocrystals are synthesized. Photoluminescence spectra and kinetics of the $\mathrm{Mn}^{2+}$ ion emission at room temperature have been studied. It is found that the quantum confinement effect does not modify the emission lifetime as compared to bulk material.

1. Introduction Doped semiconductor nanocrystals are extensively investigated to obtain basic information on impurity states in quantum dots and to examine their potential applications in novel light-emitting devices. It is well known that the quantum confinement effect modifies the electronic structure of nanocrystals when their diameter is comparable to or smaller than the diameter of the bulk exciton [1-3]. Moreover, recent results on $\mathrm{ZnS}: \mathrm{Mn}$ nanocrystals [4] show that the position of the $\mathrm{Mn}^{2+}$ emission band is slightly shifted from that of the bulk material. These authors also claim that the ${ }^{4} \mathrm{~T}_{1}$ level lifetime of manganese ions in nanocrystals is reduced by five orders of magnitude as compared to the bulk material. However, further reports did not confirm this fact [5]. Since the optical properties of semiconductor nanocrystals strongly depend on the preparation methods, sort of matrix etc., it is important to extend investigations to different manganese-doped nanocrystals incorporated in various matrices.

In this contribution we report on the synthesis of thin sol-gel film containing manganese-doped CdS nanocrystals, CdS:Mn. The time-resolved photoluminescence behavior is studied in details and a possible mechanism of radiative recombination in the presence of manganese ions is discussed.

2. Experimental Section The samples investigated were thin solid films prepared by sol-gel technique from 1-mercaptopropyltrietoxysilane (MPTS) gel containing CdS : Mn nanocrystals. First, a colloidal solution of $\mathrm{CdS}: \mathrm{Mn}$ nanocrystals was prepared by mixing two dimethylformamide (DMF) solutions at room temperature: one containing $3 \times 10^{-2} \mathrm{M}$ (mole per liter) cadmium perchlorate and $10^{-2} \mathrm{M}$ manganese perchlorate and the other containing $4 \times 10^{-2} \mathrm{M}$ sodium sulfide. The colloidal solution was purified by depositing the nanocrystals with isopropanol and redissolving in a portion of $10^{-2} \mathrm{M}$

\footnotetext{
$\left.{ }^{1}\right)$ Corresponding author; e-mail: gaponen@imaph.bas-net.by

*) Supported by Volkswagen-Stiftung and State Programs "Quantum" and "Informatics".
} 
MPTS in isopropanol, as the complexing and gelating agent. Ultrasonic treatment of the solution produces a clear viscous sol of MPTS-CdS:Mn nanocrystals. Prolonged drying of the sol on the surface of quartz at room temperature produced a thin solid film of MPTS gel with CdS:Mn nanocrystals. The average radius of nanocrystals evaluated from the energy of the absorption edge and tight-binding calculations [6] was equal to $15 \AA$.

Optical absorption spectra were examined on a Cary-500 (Varian) spectrophotometer. Photoluminescence (PL) and photoluminescence excitation (PLE) experiments were performed on a SPL 1211A (SOLAR) spectrophotometer. The typical spectral resolution in both PL and PLE experiments was 1-2 $\mathrm{nm}$. The time-resolved PL measurements were carried out using a nitrogen spark lamp with flash time of $5 \mathrm{~ns}$ as excitation source.

3. Results and Discussion The absorption spectrum of CdS : Mn nanocrystals in MPTS sol-gel film is typical for quantum-sized semiconductor nanoparticles with a blue shift of the absorption edge relative to bulk material (Fig. 1). The low concentration of $\mathrm{Mn}^{2+}$ ions does not influence the position of the band gap of CdS [7]. The PL spectrum consists of a broad emission band centered at $640 \mathrm{~nm}$.

The PL band mostly related to the intrinsic emission of manganese ions exhibits significant Stokes shift with respect to the absorption edge. The shape of the emission band does not obey a Lorentzian form. The PLE spectra show a substructure of the emission band at the short-wavelength wing, which is attributed to the contribution of intrinsic Mn luminescence and defect-related radiative recombination. The PL decay detected at various wavelengths near the maximum of the PL band is given in Fig. 2.

Time-resolved measurements revealed non-exponential decay on a millisecond scale with a noticeable contribution of submillisecond component with relative weight monotonously increased with the energy of emitted photons. Time-resolved emission spectra recovered from kinetic data indicate that the fast decay component is due to the shortwavelength wing in the emission spectrum which cannot be attributed to the transitions within $\mathrm{Mn}^{2+}$ ions (Fig. 3). As reported recently the short-time emission band is due to the recombination on deep surface traps in $\mathrm{CdS}$ nanocrystals with non-exponential decay time $[8,9]$.

We suppose, that in our CdS:Mn nanocrystals the energy transfer between manganese ions influences the PL decay since the energy transfer starts to be important for concentrations of emitting centers larger than 1\% [10]. This process is described by the

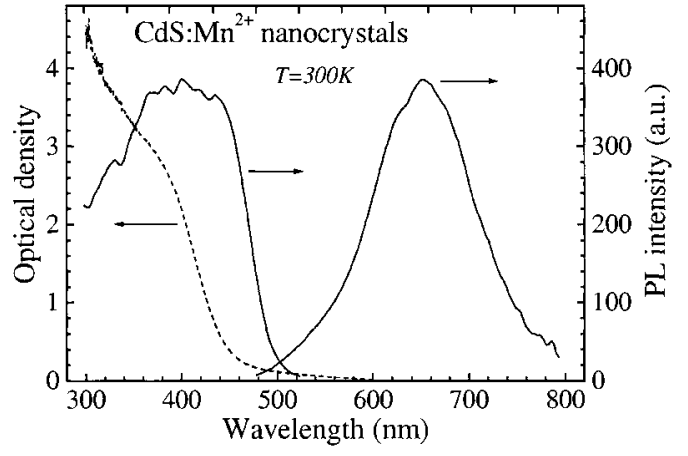

Fig. 1. Absorption (left), PLE and PL (right) spectra of CdS:Mn nanocrystals in sol-gel film at room temperature. PL was excited at $415 \mathrm{~nm}$, PLE was detected at $650 \mathrm{~nm}$ 


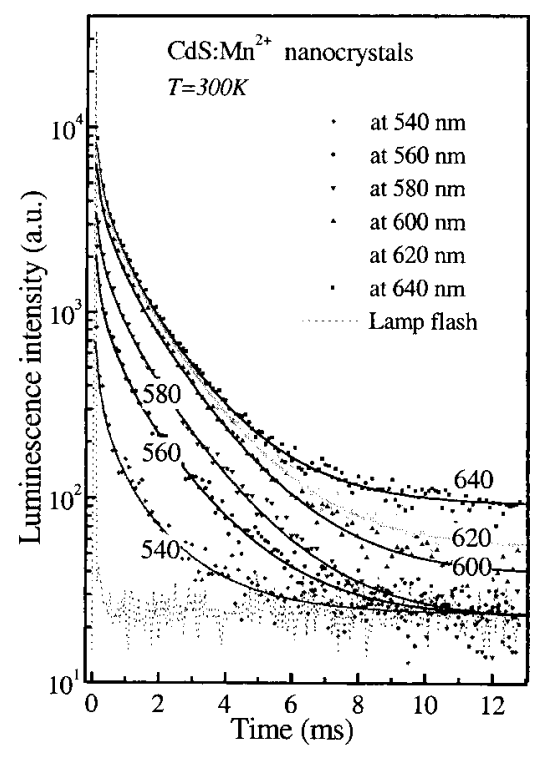

Fig. 2. Room temperature PL decay in CdS:Mn nanocrystals detected at various wavelengths. The dotted curve represents the excitation pulse. The solid lines are the Förster stretched-exponential fits with lifetime $2.3 \mathrm{~ms}$

percolation theory, and for the treatment of kinetic data it is reasonable to use the approximation by a stretched-exponential function [11],

$I(t)=I_{0} \exp \left[-t / \tau_{0}-\alpha\left(t / \tau_{0}\right)^{\beta}\right]+I_{\text {dark }}$,

where the scaling factor $\beta$ is in the range of $0.18-0.22$ and $\tau_{0}$ is the lifetime. Our samples demonstrate a constant value of $\tau_{0}=2.3 \mathrm{~ms}$ in a wide spectral range. This lifetime is likely corresponding to the emission due to the ${ }^{4} \mathrm{~T}_{1} \rightarrow{ }^{6} \mathrm{~A}_{1}$ transition in manganese ions and its value is close to that obtained by Chamarro et al. [12] in CdS:Mn nanocrystals of the same radius. Similar results were reported by Ehrlich et al. [13] for $\mathrm{Cd}_{1-x} \mathrm{Mn}_{x} \mathrm{~S}$ crystals with high $\mathrm{Mn}$ concentration $(x>0.01)$. However, PL properties of nanocrystals of semimagnetic semiconductors are expected to be influenced strongly by the preparation methods, interaction with host matrix and to differ from bulk materials [14].

The specific decay rate is to be thoroughly examined in terms of different mechanisms of energy transfer and carrier migration. Nevertheless, it is clear that taking into account the magnitude of lifetime $\tau_{0}$ which is of the same order as the bulk value, there

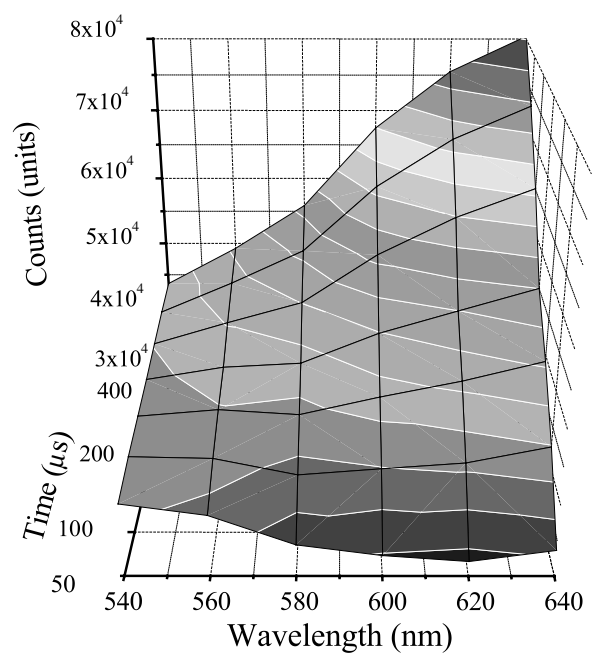
is no sign of significant enhancement of radiative decay rate in $\mathrm{Mn}$-doped nanocrystals as compared to bulk crystals and glass matrices, as it was claimed in the earlier work by Bhargava and co-workers [4].

Fig. 3. Time evolution of emission band shape of CdS:Mn nanocrystals calculated using both steady-state and time-resolved PL spectra at various excitation wavelengths 
4. Conclusions The optical properties of CdS:Mn nanocrystals incorporated in a solgel matrix are studied and PL spectra and kinetics of the $\mathrm{Mn}^{2+}$ emission at room temperature are examined in details. The manganese emission in nanocrystals is the result of the energy transfer from the semiconductor nanocrystal to the manganese ions. It is found that the quantum confinement effect does not modify the emission lifetime as compared to bulk material.

\section{References}

[1] S. V. Gaponenko, Optical Properties of Semiconductor Nanocrystals, Cambridge University Press, Cambridge 1998 (p. 245).

[2] U. Woggon, Optical Properties of Semiconductor Quantum Dots, Springer-Verlag, Berlin 1996 (p. 250).

[3] A. I. Ekimov, Al. L. Efros, and A. A. Onushchenko, Solid State Commun. 56, 921 (1985).

[4] R. N. Bhargava, D. Gallagher, X. Hong, and A. NurmikKo, Phys. Rev. Lett. 72, 416 (1994).

[5] A. A. Bol and A. Meijerink, Phys. Rev. B 58, R15997 (1998).

[6] P. E. LipPens and M. Lannoo, Phys. Rev. B 39, 10935 (1989).

[7] M. Ikeda, K. Itoh, and H. SAto, J. Phys. Soc. Jpn. 25, 455 (1968).

[8] G. Counio, S. Esnouf, T. Gacoin, and J.-P. Boilot, J. Phys. Chem. 100, 20021 (1996).

[9] F. Henneberger, J. Puls, C. Spiegelberg, A. Schülzgen, H. Rossman, V. Jungnickel, and A. L. EкIMov, Semicond. Sci. Technol. 6, A41 (1991).

[10] O. Goede and W. Heimbrodt, phys. stat. sol. (b) 146, 11 (1988).

[11] TH. Förster, Discuss. Faraday Soc. 27, 7 (1959).

[12] M. A. Chamarro, V. Voliotis, R. Grousson, P. Lavallard, T. Gacoin, G. Counio, J. P. Bollot, and R. CASES, J. Cryst. Growth 159, 853 (1996).

[13] Ch. Ehrlich, W. Busse, H. E. Gumlich, and D. Tschierse, J. Cryst. Growth 72, 371 (1985).

[14] L. Levy, N. Feltin, D. Ingert, and M. P. Pileni, J. Phys. Chem. B 101, 9153 (1997). 